Acta Crystallographica Section D

Biological

Crystallography

ISSN 1399-0047

Bing Zhang, ${ }^{a} \neq$ Caini Liu, ${ }^{b} \neq$ Wen Qian, ${ }^{\mathrm{b}}$ Yue Han, ${ }^{\mathrm{a}}$ Xiaoxia $\mathbf{L i}^{\mathbf{b}}{ }^{*}$ and Junpeng Deng ${ }^{\mathrm{a} *}$

a Department of Biochemistry and Molecular Biology, Oklahoma State University, Stillwater, OK 74078, USA, and ${ }^{\mathbf{b}}$ Department of Immunology, Lerner Research Institute, Cleveland Clinic, 9500 Euclid Avenue, Cleveland, $\mathrm{OH}$ 44195, USA

‡ These authors contributed equally.

Correspondence e-mail: lix@ccf.org, junpeng.deng@okstate.edu

\title{
Structure of the unique SEFIR domain from human interleukin 17 receptor $A$ reveals a composite ligand-binding site containing a conserved $a$-helix for Act1 binding and IL-17 signaling
}

Interleukin 17 (IL-17) cytokines play a crucial role in mediating inflammatory and autoimmune diseases. A unique intracellular signaling domain termed SEFIR is found within all IL-17 receptors (IL-17Rs) as well as the key adaptor protein Act1. SEFIR-mediated protein-protein interaction is a crucial step in IL-17 cytokine signaling. Here, the $2.3 \AA$ resolution crystal structure of the SEFIR domain of IL-17RA, the most commonly shared receptor for IL-17 cytokine signaling, is reported. The structure includes the complete SEFIR domain and an additional $\alpha$-helical C-terminal extension, which pack tightly together to form a compact unit. Structural comparison between the SEFIR domains of IL-17RA and IL-17RB reveals substantial differences in protein topology and folding. The uniquely long insertion between strand $\beta \mathrm{C}$ and helix $\alpha \mathrm{C}$ in IL-17RA SEFIR is mostly well ordered, displaying a helix $\left(\alpha \mathrm{CC}_{\text {ins }}^{\prime}\right)$ and a flexible loop $\left(\mathrm{CC}^{\prime}\right)$. The $\mathrm{DD}^{\prime}$ loop in the IL-17RA SEFIR structure is much shorter; it rotates nearly $90^{\circ}$ with respect to the counterpart in the IL-17RB SEFIR structure and shifts about $12 \AA$ to accommodate the $\alpha \mathrm{CC}_{\text {ins }}^{\prime}$ helix without forming any knots. Helix $\alpha \mathrm{C}$ was identified as critical for its interaction with Act1 and IL-17-stimulated gene expression. The data suggest that the heterotypic SEFIR-SEFIR association via helix $\alpha \mathrm{C}$ is a conserved and signature mechanism specific for IL-17 signaling. The structure also suggests that the downstream motif of IL-17RA SEFIR together with helix $\alpha \mathrm{C}$ could provide a composite ligand-binding surface for recruiting Act1 during IL-17 signaling.

\section{Introduction}

Members of the IL-17 cytokine family (IL-17A to IL-17F) are important regulators of both innate and adaptive immune responses. However, the strong inflammatory response overstimulated by IL-17 contributes to the pathogenesis of various human and animal diseases, such as rheumatoid arthritis, multiple sclerosis (MS), experimental autoimmune encephalomyelitis (EAE; a mouse model of MS) and allergen-induced pulmonary inflammation (Ye et al., 2001; Nakae et al., 2002; Iwakura \& Ishigame, 2006; Schnyder-Candrian et al., 2006; Conti et al., 2009; Gonzalez-García et al., 2009; Hu et al., 2011). The IL-17 receptor (IL-17R) family comprises five members (IL-17RA to IL-17RE) with considerable sequence divergence, which are all membrane-bound proteins containing single transmembrane domains. IL-17Rs contain fibronectin type III (FnIII) domains within the extracellular portion and a unique structural motif within the cytoplasmic tails termed the SEFIR [SEF (similar expression to fibroblast growth factor genes) and IL-17R] domain (Novatchkova et al., 2003). SEFIR domains share limited homology with the Toll/IL-1
Received 20 January 2014

Accepted 6 March 2014

PDB reference: IL-17 receptor A SEFIR domain, 4nux 
Table 1

Statistics of data collection and refinement.

Values in parentheses are for the highest resolution shell.

\begin{tabular}{lll}
\hline & Se-SAD & Native \\
\hline $\begin{array}{l}\text { Data collection } \\
\text { Beamline }\end{array}$ & 19-ID, APS & 19-ID, APS \\
Wavelength $(\AA)$ & 0.97935 & 0.97935 \\
Space group & $C 222_{1}$ & $C 222_{1}$ \\
Unit-cell parameters $(\AA)$ & $a=75.9, b=133.8$, & $a=76.2, b=136.1$, \\
& $c=56.2$ & $c=55.4$ \\
Resolution $(\AA)$ & $50-3.0$ & $50-2.3$ \\
Total reflections & 42331 & 87400 \\
Unique reflections & 6057 & 13104 \\
$R_{\text {merge }}(\%)$ & $15.0(78.3)$ & $12.4(65.8)$ \\
Multiplicity & 7.0 & 6.7 \\
Completeness (\%) & $99.9(100)$ & $98.0(87.3)$ \\
$\langle I / \sigma(I)\rangle$ & $12.8(2.0)$ & $15.3(1.6)$ \\
Refinement & & \\
Resolution range used $(\AA)$ & & $33.2-2.3$ \\
No. of reflections used & & 12124 \\
$R_{\text {work }} / R_{\text {free }}(\%)$ & & $18.9 / 24.1$ \\
R.m.s. deviations & & \\
$\quad$ Bond lengths $(\AA)$ & & 0.008 \\
Bond angles $\left({ }^{\circ}\right)$ & & 1.066 \\
Ramachandran values $(\%)$ & & 96.3 \\
$\quad$ Preferred regions & & 3.7 \\
Allowed regions & & \\
\hline
\end{tabular}

$\dagger R_{\text {merge }}=\sum_{h k l} \sum_{i}\left|I_{i}(h k l)-\langle I(h k l)\rangle\right| / \sum_{h k l} \sum_{i} I_{i}(h k l) . \quad \neq R_{\text {work }}=\sum_{h k l}|| F_{\text {obs }}|-| F_{\text {calc }}|| /$ $\sum_{h k l}\left|F_{\text {obs }}\right| ; R_{\text {free }}$ was calculated using $5 \%$ of the data.

receptor (TIR) domains of Toll-like receptors (TLRs) and IL-1R (Novatchkova et al., 2003). Proteins containing SEFIR and TIR domains together constitute the STIR (SEFIR/TIR) domain superfamily. NF- $\kappa \mathrm{B}$ activator 1 [Act1; also known as connection to $\mathrm{I}-\kappa \mathrm{B}$ kinase and stress-activated protein kinase (CIKS); Leonardi et al., 2000] is also a member of the SEFIR protein family and is an essential component in the signaling of several IL-17 cytokines (Chang et al., 2006; Qian et al., 2007; Claudio et al., 2009; Swaidani et al., 2009). Although the exact stoichiometry of the complex of IL-17R and Act1 in IL-17 signaling is not known, SEFIR-mediated homotypic and heterotypic interactions among IL-17Rs and Act1 play a crucial role in IL-17R signaling. The crystal structure of the IL-17RB SEFIR domain has recently been determined, which revealed a unique knot in protein folding (Zhang et al., 2013). Structural elements unique to SEFIR and distinct from TIR domains have been identified, including a long $\mathrm{CC}^{\prime}$ insertion (between strand $\beta \mathrm{C}$ and helix $\alpha \mathrm{C}$ ) and a rigid short helix $\alpha \mathrm{B}^{\prime}$. It has been shown that the $\alpha \mathrm{C}$ helices of the SEFIR domains from IL-17RB SEFIR and Act1 are critical for their heterotypic interactions, while helix $\alpha \mathrm{B}^{\prime}$ of Act1 is responsible for its self-association. The study provided the first structural view of the IL-17 receptor intracellular signaling, unraveling the mechanism for the specificity of the SEFIR versus the TIR domain in their respective signaling pathways. However, questions still remain for the SEFIR domains from other IL-17 receptors owing to low pairwise sequence identity (less than $20 \%$ ). In particular, IL-17RA appears to be unique among all IL-17Rs and contains the longest intracellular domain, which extends well beyond its SEFIR domain. In addition, it has been shown that IL-17RA signaling requires not only its
SEFIR domain but also a short C-terminal extension of about 100 amino acids (Onishi et al., 2010). This tandem structural motif was termed the SEFEX domain and was found to be important for both binding Act1 and IL-17 cytokine signaling (Onishi et al., 2010). The fact that IL-17RA SEFIR alone is not sufficient for binding Act1 and IL-17 signaling suggests that it could be structurally unique when compared with the SEFIR domains of other IL-17Rs and Act1.

Since IL-17 is an important regulator for inflammation, blocking IL-17 signaling is a promising approach for the treatment of autoimmune diseases. For example, brodalumab, an antibody against IL-17RA for the treatment of psoriasis, is currently in phase 2 clinic trials (Papp et al., 2012). Given the importance of SEFIR-SEFIR interactions in IL-17 cytokine signaling, a better mechanistic understanding of these interactions is crucial for the development of new and improved therapeutics for the treatment of IL-17 cytokine-mediated inflammatory diseases. Here, we report the crystal structure of a fragment from the IL-17RA intracellular signaling domain including its SEFIR domain and a short C-extension at $2.3 \AA$ resolution. The structure displays large differences in protein topology and conformation with respect to the structure of IL-17RB SEFIR. Our structural and functional analyses again reveal that helix $\alpha \mathrm{C}$ of IL-17RA is important for its binding to Act1 and IL-17 cytokine signaling. This suggests that the heterotypic SEFIR-SEFIR association via helix $\alpha \mathrm{C}$ is a conserved and signature mechanism specific for IL-17 cytokine signaling. The structure of IL-17RA SEFIR also suggests that the downstream motif of IL-17RA SEFIR could fold back close to helix $\alpha \mathrm{C}$ of SEFIR, together providing a composite ligand-binding surface that is necessary for recruiting Act1 during IL-17 cytokine signaling.

\section{Materials and methods}

\subsection{Protein expression and purification}

A fragment of human IL-17RA (amino-acid residues 351597; termed IL-17RA SEFIR) containing the entire SEFIR domain was cloned into a modified pET-28 vector with an $\mathrm{N}$-terminal $6 \times$ His tag. The IL-17RA SEFIR domain was expressed and purified using a similar protocol as described previously (Krumm et al., 2008). The N-terminal $6 \times$ His tag was removed using Tobacco etch virus (TEV) protease. The purified protein was concentrated to $20 \mathrm{mg} \mathrm{ml}^{-1}$ and crystallized in a condition consisting of 0.1 $M$ HEPES pH 7.5, $3.5 M$ sodium nitrate after about a week at room temperature. However, the crystals did not diffract at the synchrotron. The purified protein was then subjected to limited proteolysis by treatment with chymotrypsin, and was subsequently further purified by size-exclusion chromatography on a Superdex S200 column (GE Healthcare). The purified chymotrypsinprocessed protein sample displayed a single peak on the S200 sizing profile with an estimated molecular mass of $28 \mathrm{kDa}$, which is nearly the same as the intact protein sample. Selenomethionine (SeMet)-substituted IL-17RA SEFIR domain was expressed in M9 minimal medium supplemented with amino 
acids as described by Van Duyne et al. (1993) and purified similarly to the native protein using the procedures described above. For optimal reproducibility of crystallization, all purified proteins were flash-frozen and stored at $-80^{\circ} \mathrm{C}$ until usage (Deng et al., 2004).

\subsection{Crystallization, data collection and structure determination}

The chymotrypsin-treated IL-17RA SEFIR crystallized in a condition consisting of $0.1 M$ MES pH $6.0,5-10 \%$ PEG $10000.20 \%$ glycerol was added to the mother liquor as a cryoprotectant. A set of data was collected from an SeMetsubstituted protein crystal on beamline 19-ID at the Advanced Photon Source, Argonne National Laboratory, USA. The structure was solved by the single-wavelength anomalous

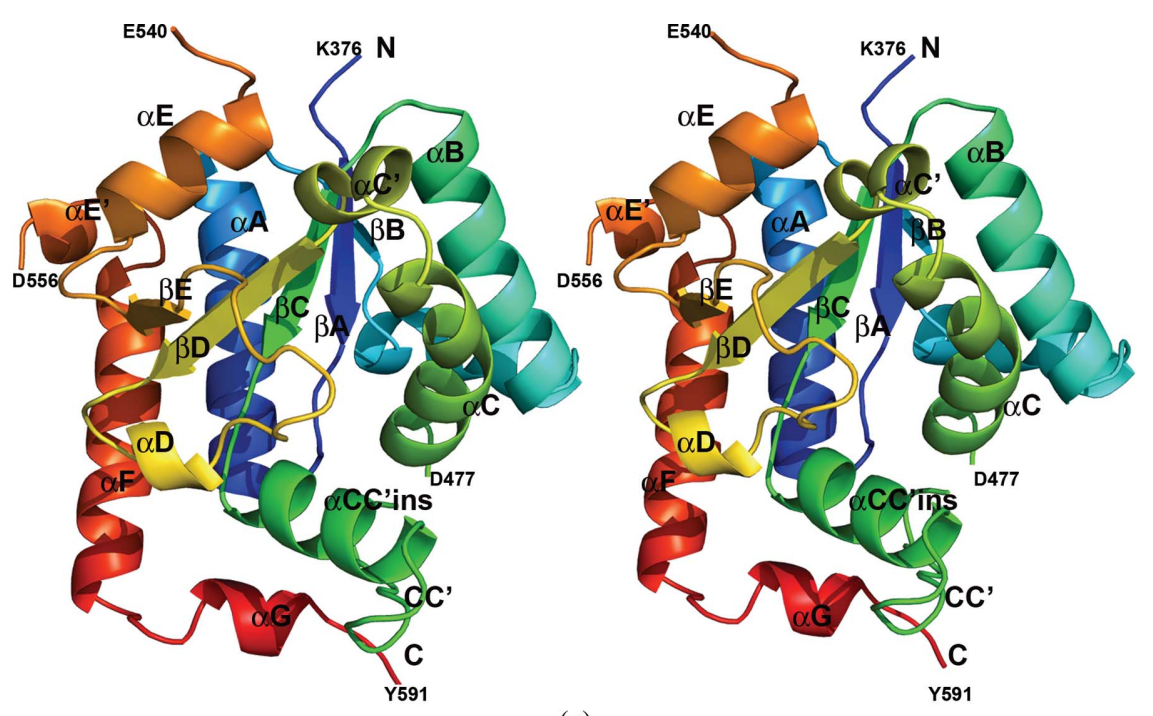

(a)
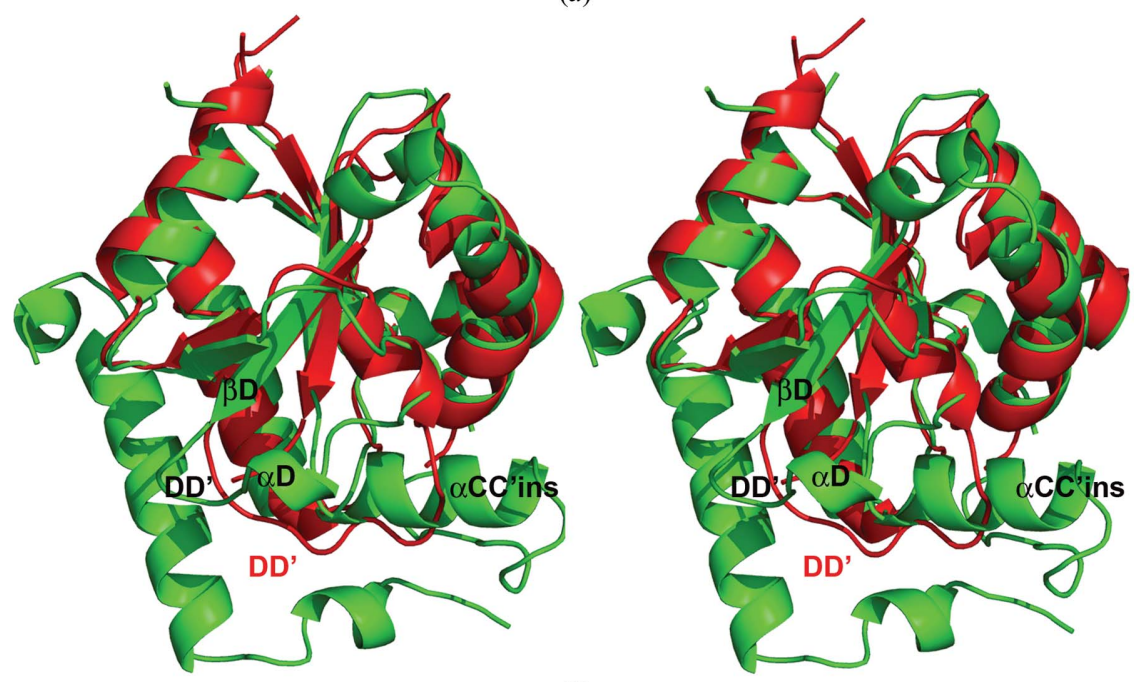

(b)

Figure 1

The unique structure of IL-17RA SEFIR. (a) Stereoview of the IL-17RA SEFIR domain. The secondary structures are shown as rainbow-colored ribbons and are labeled. (b) Pairwise comparison with IL-17RB SEFIR. Depicted is a stereoview of the superimposed structures of the SEFIR domains from IL-17RA (green) and IL-17RB (red). The secondary structures with the greatest topological and conformational differences are labeled. dispersion method using HKL-3000 (Minor et al., 2006). A nearly complete model was constructed from the experimental phases obtained from the SeMet crystal data. This model was used to solve the native structure by the molecular-replacement method using Phaser (McCoy et al., 2007). PHENIX (Adams et al., 2010) was used for refinement and Coot (Emsley et al., 2010) was used for iterative manual model building. Translation, libration and screw-rotation displacement (TLS) groups used in the refinement were defined by the TLMSD server (Painter \& Merritt, 2006). The current model has good geometry and refinement statistics (Table 1). The final structure was refined to $2.3 \AA$ resolution and the crystallographic $R_{\text {work }}$ and $R_{\text {free }}$ values are 18.9 and $24.1 \%$, respectively. The final protein model has $96.3 \%$ of all residues residing in the most favored region of the Ramachandran plot and $3.7 \%$ in additionally allowed regions, as calculated by the MolProbity server (Chen et al., 2010). All molecular-graphics figures were generated with PyMOL (DeLano, 2002). Atomic coordinates and structure factors have been deposited in the Protein Data Bank (http://www.rcsb.org; PDB code 4nux).

\subsection{Cell culture, plasmids and antibodies}

Mouse embryonic fibroblasts (MEFs) were maintained in Dulbecco's modified Eagle's medium (DMEM) supplemented with $10 \%$ fetal bovine serum (FBS), penicillin $\mathrm{G}\left(100 \mu \mathrm{g} \mathrm{ml}^{-1}\right)$, and streptomycin $\left(100 \mu \mathrm{g} \mathrm{ml}^{-1}\right)$. Complementary DNA (cDNA) encoding Myc-tagged mouse IL17RA and its point mutants were subcloned into the plasmid pMSCV-IRES-GFP. Mouse Act1 antibody was generated by Invitrogen custom antibody services. Mouse Myc antibody was purchased from Cell Signaling.

\subsection{Retroviral infection and co-immuno- precipitation}

For reconstitution assays in MEFs, cells were infected by retroviral supernatant as described previously (Qian et al., 2007). For co-immunoprecipitations, cell extracts were incubated with antibody $(1 \mu \mathrm{g})$ and protein A beads $(20 \mu \mathrm{l})$. After overnight incubation, beads were washed four times with lysis buffer, resolved by SDS-PAGE, and analyzed by Western blotting according to standard procedures.

\subsection{Real-time polymerase chain reaction assays}

Total RNA was extracted with TRIzol reagent according to the manufacturer's instructions. The cDNAs were synthesized 
and real-time reverse transcription polymerase chain reaction (RT-PCR) assays were performed as described previously (Qian et al., 2007).

\section{Results}

\subsection{The structure of IL-17RA SEFIR}

The structure of IL-17RA SEFIR displays a compact globular architecture comprised of a five-stranded $(\beta \mathrm{A}-\beta \mathrm{E})$ parallel $\beta$-sheet that is wrapped by 11 helices $\left(\alpha \mathrm{A}, \alpha \mathrm{B}^{\prime}, \alpha \mathrm{B}\right.$, $\alpha \mathrm{CC}_{\mathrm{ins}}^{\prime}, \alpha \mathrm{C}, \alpha \mathrm{C}^{\prime}, \alpha \mathrm{D}, \alpha \mathrm{E}, \alpha \mathrm{E}^{\prime}, \alpha \mathrm{F}$ and $\alpha \mathrm{G}$; Fig. $\left.1 a\right)$ with loops of various lengths connecting the secondary structures (the individual loop that connects the $\beta$-strand and its next neighboring helix is named after the names of the secondary structures, i.e. the $\mathrm{DD}^{\prime}$ loop connects strand $\beta \mathrm{D}$ and helix $\alpha \mathrm{D}$ ). The structure is well defined except for two flexible loops, the electron densities of which are not observable. One is a sixamino-acid short linker (residues 471-476) within the $\mathrm{CC}^{\prime}$ loop. The other is a 14-amino-acid linker (residues 541-555) connecting helices $\alpha \mathrm{E}$ and $\alpha \mathrm{E}^{\prime}$. Besides the complete SEFIR domain (residues 376-540) of IL-17RA, the structure reported here additionally includes a short C-terminal extension (residues 556-591) of about 35 amino acids. Extending from the C-terminus of the SEFIR domain, the C-terminal fragment (residues 556-591) forms additional $\alpha$-helices $\left(\alpha \mathrm{E}^{\prime}, \alpha \mathrm{F}\right.$ and $\alpha \mathrm{G}$; Fig. 1a). The longer helix $\alpha \mathrm{F}$ is sandwiched by two short helices, $\alpha \mathrm{E}^{\prime}$ and $\alpha \mathrm{G}$, in a Z-shaped crank shape. This predominantly helical C-extension of SEFIR packs tightly against helices $\alpha \mathrm{A}$ and $\mathrm{CC}_{\mathrm{ins}}^{\prime}$ and loops $\mathrm{CC}^{\prime}, \mathrm{DD}^{\prime}$ and $\mathrm{EE}^{\prime}$ through

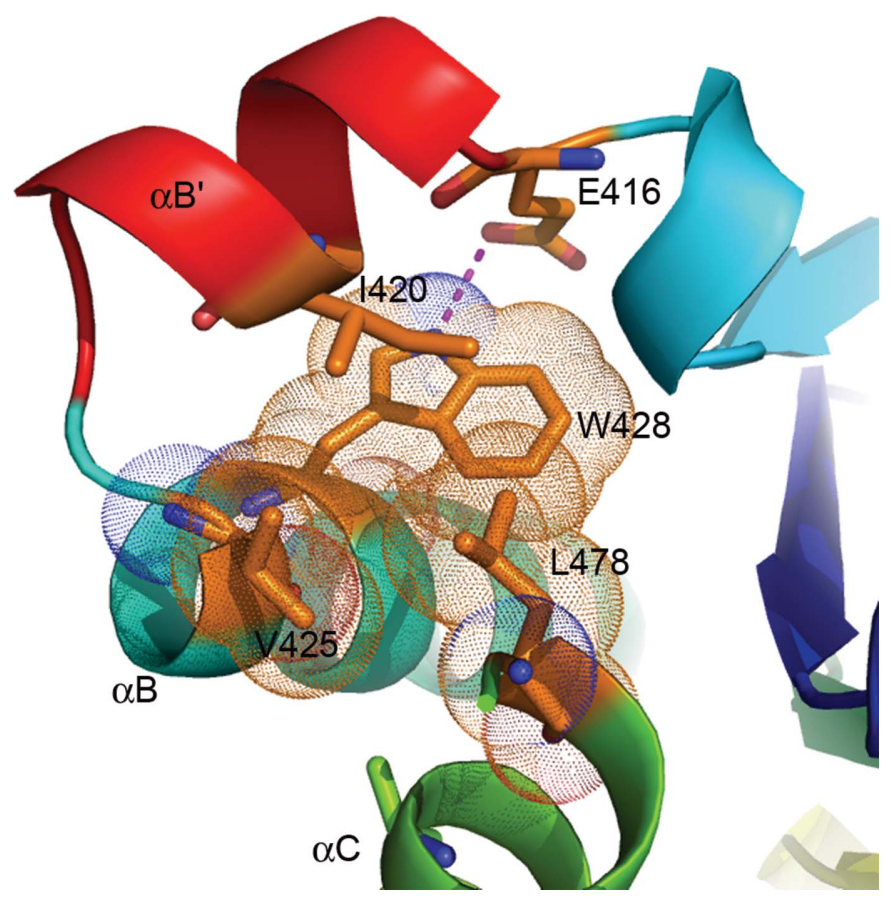

Figure 2

Helix $\alpha \mathrm{B}^{\prime}$ is tethered to helices $\alpha \mathrm{B}$ and $\alpha \mathrm{C}$ via hydrophobic and hydrogen-bonding interactions. The side chains of the hydrophobic residues are shown as sticks with dotted spheres showing the van der Waals radius for each atom. The hydrogen bond between $\mathrm{N}^{\varepsilon 1}$ of Trp428 and $\mathrm{O}^{\varepsilon 1}$ of Glu416 is shown as a purple dashed line. extensive van der Waals interactions, stabilizing the overall architecture.

\subsection{IL-17RA SEFIR versus IL-17RB SEFIR}

The structure of IL-17RA SEFIR partially resembles that of IL-17RB SEFIR (Zhang et al., 2013), with an r.m.s.d. of $1.82 \AA$ over 121 aligned $\mathrm{C}^{\alpha}$ atoms (Fig. $1 b$ ). The central five-stranded parallel $\beta$-sheets of the two structures superimpose very well with little conformational change. Helices $\alpha \mathrm{A}, \alpha \mathrm{B}^{\prime}, \alpha \mathrm{B}, \alpha \mathrm{C}$ and $\alpha \mathrm{E}$ also align very well between the two structures. Similar to the observation in the structure of IL-17RB SEFIR, the structure of IL-17RA SEFIR also contains a short helix $\alpha \mathrm{B}^{\prime}$ (residue 413-422) that links helix $\alpha \mathrm{B}$ and strand $\beta \mathrm{B}$ and is nearly perpendicular to helix $\alpha \mathrm{B}$ (Fig. 1). Helix $\alpha \mathrm{B}^{\prime}$ is also tethered to helices $\alpha \mathrm{B}$ and $\alpha \mathrm{C}$ through hydrophobic interactions in a similar way as observed in the IL-17RB SEFIR structure. Specifically, residue Ile 420 on helix $\alpha \mathrm{B}^{\prime}$ is associated with a hydrophobic platform comprised of residues Val425 and $\operatorname{Trp} 428$ on helix $\alpha \mathrm{B}$ and Leu478 on helix $\alpha \mathrm{C}$ (Fig. 2). In addition, residue Trp 428 on helix $\alpha \mathrm{B}$ is hydrogen bonded to Glu416 on $\alpha \mathrm{B}^{\prime}$, which further stabilizes and locks the $\alpha \mathrm{B}^{\prime}$ and $\alpha \mathrm{B}$ helices into a rigid conformation. Despite these structural similarities, the two SEFIR structures also display significant differences in protein folding and conformation. In the IL17RA SEFIR structure there is a short helix $\left(\alpha \mathrm{C}^{\prime}\right.$; residues 493-498) between the kinked helix $\alpha \mathrm{C}$ and strand $\beta \mathrm{D}$ (Figs. $1 a$ and 3). The equivalent part in the IL-17RB SEFIR structure is a loop. In contrast to the complete disorder in the structure of IL-17RB SEFIR, the unique and long insertion between strand $\beta \mathrm{C}$ and helix $\alpha \mathrm{C}$ in the IL-17RA SEFIR structure is mostly well ordered, displaying a helix $\left(\alpha \mathrm{CC}_{\text {ins }}^{\prime}\right)$ and a flexible loop (CC') (Figs. 1 and 3). This observation is consistent with our secondary-structure prediction (Zhang et al., 2013). Helix $\alpha \mathrm{CC}_{\text {ins }}^{\prime}$ is nearly perpendicular to both strand $\beta \mathrm{C}$ and helix $\alpha \mathrm{C}$ in the IL-17RA SEFIR structure. The most striking observation when comparing the two SEFIR structures is the absence of the knot feature in IL-17RA SEFIR (Fig. 3). There is a large conformational difference in the secondary structures connecting strands $\beta \mathrm{D}$ and $\beta \mathrm{E}$ between the two crystal structures. In the IL-17RA SEFIR structure the much shorter DD' loop together with the short helix $\alpha \mathrm{D}$ rotate up almost $90^{\circ}$ with respect to the much longer $\mathrm{DD}^{\prime}$ loop in IL-17RB SEFIR structure and shift about $12 \AA$ to accommodate the $\alpha \mathrm{CC}_{\text {ins }}^{\prime}$ helix (Figs. $1 b$ and 3). As a consequence, the topology of IL-17RA SEFIR is completely different from that of IL17RB SEFIR and lacks a knot. In addition, the structure of IL-17RB SEFIR does not contain any C-terminal extensions beyond helix $\alpha \mathrm{E}$ as observed in the IL-17RA SEFIR structure (Fig. 1).

\subsection{Helix $a$ C is critical for IL-17RA signaling}

The adaptor protein Act1 is a key component in IL-17 cytokine signaling (Chang et al., 2006, 2011; Qian et al., 2007; Claudio et al., 2009; Swaidani et al., 2009; Ramirez-Carrozzi et al., 2011; Song et al., 2011). It has been implied that IL-17RA may be a common receptor unit for IL-17 cytokines. Act1 
mediates IL-17RA signaling in a SEFIR-dependent manner (Claudio et al., 2009; Swaidani et al., 2009) via the heterodimerization of Act1 and IL17RA. Deletion of the SEFIR domain of either protein abolished their interaction. To identify residues in IL-17RA SEFIR that are key to its function, based on the structure of IL-17RA SEFIR we generated five mutants of mouse IL-17RA SEFIR and performed reconstitution and co-immunoprecipitation experiments in IL-17RA ${ }^{-1-}$ MEFs. Since we have previously shown that helix $\alpha \mathrm{C}$ of the SEFIR domains of IL-17RB and Act1 is important for SEFIR-mediated protein-protein interactions and IL-17 signaling (Liu et al., 2011; Zhang et al., 2013), we decided to test surface residues of IL-17RA SEFIR located on the secondary structures that are in the vicinity of helix $\alpha \mathrm{C}$. The mutations are grouped into the following four regions on the surface of IL-17RA SEFIR (Fig. 4): $\alpha \mathrm{C}$, M1 (T483A/N488A/M489A); $\alpha$ CC $_{\text {ins }}^{\prime}$, M2 (I460A/L461A) and M3 (R451A/ $\mathrm{Q} 454 \mathrm{~A} / \mathrm{K} 458 \mathrm{~A}) ; \quad \alpha \mathrm{B}^{\prime}, \quad \mathrm{M} 4 \quad(\mathrm{E} 419 \mathrm{~A} /$ Q420A/E424A); $\alpha$ B, M5 (V427A/M428A). We found that only mutations in helix $\alpha \mathrm{C}$ (M1; T483A/N488A/M489A) substantially decreased the interaction of IL-17RA with Act1 and abolished IL-17A-induced gene expression (Figs. $4 a$ and 4b). In contrast, all other mutations had little impact on the interaction of IL-17RA with Act1 and IL-17A signaling. The results here implicate that the $\alpha \mathrm{C}$ helix of IL-17RA is the most critical region within the SEFIR domain for its heterotypic interaction with Act1 and is essential for IL-17A signaling.

\section{Discussion}

We have determined the $2.3 \AA$ resolution crystal structure of the SEFIR domain of IL-17RA, the most commonly shared receptor subunit for IL-17 cytokine-mediated immune responses. The current study provides key information on the unique structural module that is the signature for IL-17 intracellular signaling. The structure of IL-17RA SEFIR revealed substantial differences in protein folding and conformation when compared with the recently reported structure of IL-17RB SEFIR (Zhang et al., 2013). Our structural and functional analysis of IL-17RA SEFIR identified helix $\alpha \mathrm{C}$ as a critical structural motif for heterotypic SEFIRSEFIR interactions between Act1 and IL-17RA. Specifically, we show that alanine substitutions of residues Thr483, Asn488 and Met489 that are located on the surface of helix $\alpha \mathrm{C}$ of mouse IL-17RA SEFIR impaired its interaction with Act1 as well as IL-17A-induced gene expression. Our data suggest that helix $\alpha \mathrm{C}$ is a conserved hot spot for heterotypic SEFIR- mediated protein interactions. The structure of IL-17RA SEFIR reveals that its structural stability is dependent on the helical C-extension, which is a distinct feature specific to IL-17RA. The structure also suggests that a structural motif further downstream of SEFIR could cluster spatially with helix $\alpha \mathrm{C}$ and provide a composite surface for recruiting Act1 during IL-17 signaling.

The crystal structure of IL-17RB SEFIR has recently been reported, which provided a fresh look at the unique signaling module responsible for IL-17 signaling and provided important structural insights (Zhang et al., 2013). However, the low pairwise sequence identity (less than 20\%) among IL-17R SEFIR domains warrants further structural and functional studies on other SEFIR domains. This is particularly important for IL-17RA SEFIR because it is shared among essentially all IL-17 receptor complexes for signaling (Gaffen, 2009; Chang \& Dong, 2011). Comparison of the structures of the IL-17RA and IL-17RB SEFIR domains revealed that both SEFIR domains share a conserved set of secondary structures, especially the central five-stranded parallel $\beta$-sheet and helices $\alpha \mathrm{A}, \alpha \mathrm{B}^{\prime}, \alpha \mathrm{B}, \alpha \mathrm{C}$ and $\alpha \mathrm{E}$, forming a compact structural core (Fig. 1b). Similar to our observations for the structure of IL17RB SEFIR, the short helix $\alpha \mathrm{B}^{\prime}$ between $\beta \mathrm{B}$ and $\alpha \mathrm{B}$ is also tethered to helices $\alpha \mathrm{B}$ and $\alpha \mathrm{C}$ through hydrophobic interactions and a conserved hydrogen bond between Trp428 and Glu416 in the IL-17RA SEFIR structure. These observations suggest that helix $\alpha \mathrm{B}^{\prime}$ adopts a stable and relatively rigid conformation that distinguishes the SEFIR domain from a TIR domain (Zhang et al., 2013). We previously showed that 
helix $\alpha \mathrm{B}^{\prime}$ of Act1 was not essential for its heterotypic SEFIRSEFIR interactions, since deletion of this segment in Act1 did not have a significant impact on its interaction with IL-17RA (Liu et al., 2011). However, we showed that helix $\alpha \mathrm{B}^{\prime}$ in Act1 SEFIR is instead essential for its homotypic SEFIR-SEFIR interactions, since deletion of helix $\alpha \mathrm{B}^{\prime}$ in the Act1 SEFIR domain greatly reduced its self-association and IL-17 cytokine-mediated gene expression (Zhang et al., 2013). The functional role of helix $\alpha \mathrm{B}^{\prime}$ in IL-17RA SEFIR is not clear. However, alanine substitutions of charged and polar residues on the surface of helix $\alpha \mathrm{B}^{\prime}$ did not show any significant impact on its binding to Act1 and IL-17A signaling (Figs. $4 a$ and $4 b$ ). Therefore, helix $\alpha \mathrm{B}^{\prime}$ seems to represent a conserved structural feature among all SEFIR-containing proteins, but may possess varying functional roles. In addition, we have previously shown helix $\alpha \mathrm{C}$ of both Act1 and IL-17RB SEFIR domains to be a key structural element and a 'hot spot' for heterotypic SEFIR-mediated protein interactions. Surface mutations on helix $\alpha \mathrm{C}$ in both the Act1 and IL-17RB SEFIR domains disrupted their respective interactions with IL-17RA and

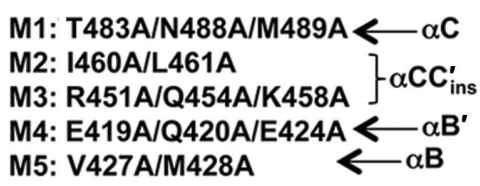

M5: V427A/M428A

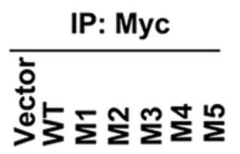

IB $\left.\right|_{\text {RA-Myc }} ^{\text {Act1 }}$
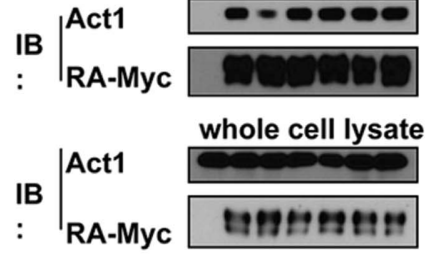

\begin{tabular}{|c|c|c|c|c|c|c|c|}
\hline Mutant & Vector & WT & M1 & M2 & M3 & M4 & M5 \\
\hline $\begin{array}{c}\text { Act 1 } \\
\text { binding }\end{array}$ & 0 & 100 & 31 & 102 & 107 & 107 & 98 \\
\hline
\end{tabular}

(a)

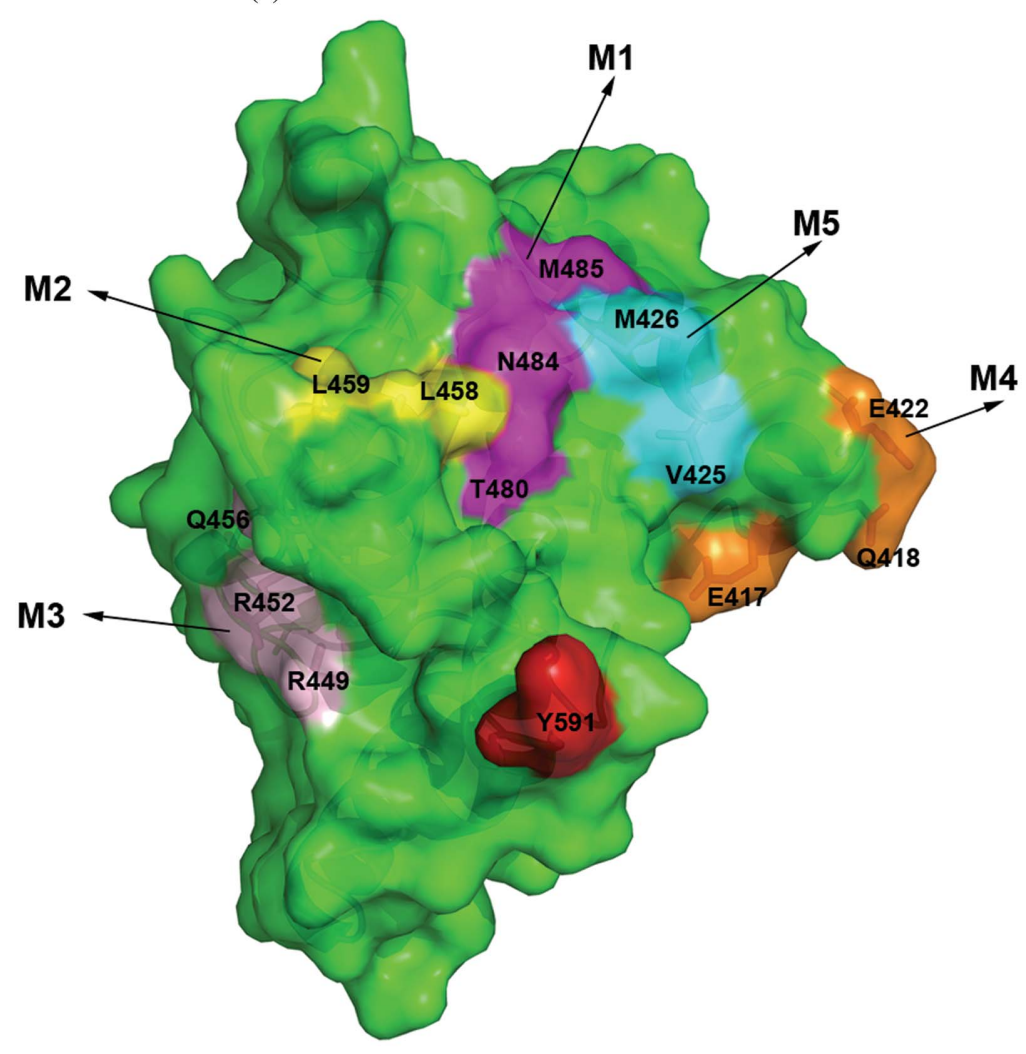

(c)

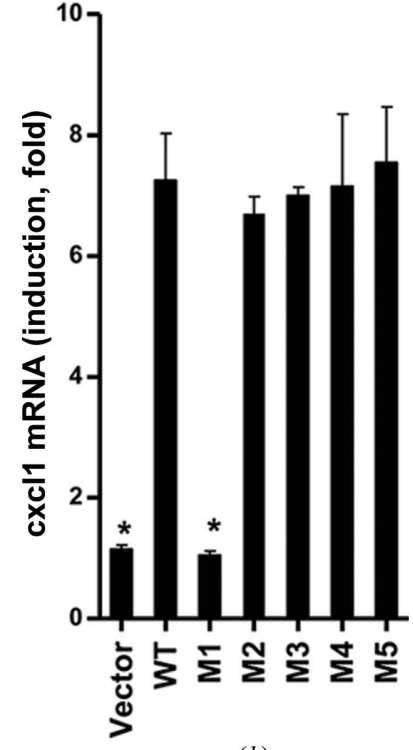

(b)
Act1, and abolished IL-17 cytokinestimulated gene expression (Liu et al., 2011; Zhang et al., 2013). This led to our hypothesis that heterotypic SEFIRSEFIR association via helix $\alpha \mathrm{C}$ is a conserved and signature mechanism specific for IL-17R signaling. The structural and functional data presented here on IL-17RA SEFIR support this hypothesis.

Despite sharing many structural features with IL-17RB SEFIR, the IL17RA SEFIR structure displays substantial differences in protein folding and conformation. First of all, IL-17RA SEFIR is topologically different from IL-17RB SEFIR and does not contain any knot features.

\section{Figure 4}

Helix $\alpha$ C of IL-17RA SEFIR is key to function. (a) IL-17RA-deficient MEFs were reconstituted with empty vector and Myc-tagged mouse RA mutants by retroviral infection, after which the cells were treated with IL-17 for $10 \mathrm{~min}$ followed by immunoprecipitation with antiMyc antibody and Western blot analysis with the indicated antibodies. The densities of Act1 bands from the immunocomplexes and whole cell lysate were quantified using ImageJ and their ratios were normalized with respect to the wild-type sample and shown as Act1 binding. (b) The same cells as in $(a)$ were treated with IL-17 for $3 \mathrm{~h}$. The abundances of $c x c l 1$ mRNAs were measured by real-time RT-PCR and the induction (fold) of mRNA was calculated as the ratio of the amount of mRNA in the treated sample compared with that in the untreated sample. Data are means \pm SEMs from three experiments. An asterisk indicates $p<0.05$ (a difference from the wild-type sample). (c) The functionally defective mutations are located on the surface of helix $\alpha \mathrm{C}$. The IL-17RA SEFIR structure is shown in green as a surface representation. The surfaces of the human IL-17RA SEFIR structure corresponding to the five mouse mutation sites are colored magenta (M1; T480/N484/M485), yellow (M2; L458/L459), light pink (M3; R449/R452/Q456), orange (M4; E417/Q418/E422) and cyan (M5; V425/M426). The C-terminal end of helix $\alpha \mathrm{G}$ (residue Tyr591) is shown in red. Note that Tyr591 is oriented in the same direction as the surface of helix $\alpha \mathrm{C}$. 
Secondly, the unique signature insertion between $\beta \mathrm{C}$ and $\alpha \mathrm{C}$ is well ordered in the IL-17RA SEFIR structure, in comparison to the complete disorder in the IL-17RB SEFIR structure that is presumably owing to significant flexibility. Although our mutagenesis studies on the surface of helix $\alpha \mathrm{CC}_{\mathrm{ins}}^{\prime}$ showed negligible effects on the binding to Act1 and IL-17-stimulated gene expression, $\alpha \mathrm{CC}_{\mathrm{ins}}^{\prime}$ is tightly sandwiched between helices $\alpha \mathrm{C}$ and $\alpha \mathrm{D}$ and contributes to the overall structural integrity of IL-17RA SEFIR (Figs. $1 a$ and 3). Besides the drastic difference in topology, the greatest conformational changes observed between the two SEFIR structures lie in the DD' loop and helix $\alpha \mathrm{D}$. The $\mathrm{DD}^{\prime}$ loop in IL-17RA SEFIR is much shorter compared with that in IL-17RB SEFIR. Together with the short helix $\alpha \mathrm{D}$, it rotates almost $90^{\circ}$ with respect to the IL-17RB SEFIR structure and shifts by more than $12 \AA$, making sufficient space to accommodate the $\alpha \mathrm{CC}_{\text {ins }}^{\prime}$ helix.

IL-17RA is unique among all IL-17Rs in containing the longest intracellular domain, which extends well beyond the SEFIR domain. Although the SEFIR domain of IL-17RA is critical for function, it has also been shown that its C-terminal extension of about 100 amino acids is additionally required for complete Act1 binding and IL-17 signaling (Onishi et al., 2010). This tandem module was termed the SEFEX domain and is uniquely present in the IL-17RA intracellular domain. The fact that IL-17RA SEFIR alone is not sufficient for binding Act1 and for IL-17 signaling suggests that it could be structurally unique when compared with the SEFIRs from other IL17Rs and Act1. Indeed, the IL-17RA SEFIR structure contains not only the complete SEFIR domain but also a short helical C-terminal extension. The additional helices from the C-terminal extension of the SEFIR are tethered to the protein core and provide important stabilization for the structural folding. Therefore, the SEFIR domain of IL-17RA itself is structurally and functionally distinct compared with other SEFIRs lacking C-terminal extensions. Onishi and coworkers showed that a downstream region between residues 625 and 645 is also critical for IL-17 signaling, and named the tandem of SEFIR and its C-terminal extension the SEFEX domain. Although the structure of IL-17RA SEFIR does not extend beyond residue 591, it provides insights into a possible role of the SEFEX domain. Specifically, the C-terminal helix $\alpha \mathrm{G}$ is pointing towards the same functional surface of $\alpha \mathrm{C}$ (Figs. $1 a$ and $4 c$ ), mutations in which abolished the heterotypic interactions between IL-17RA and Act1 as well as IL-17stimulated gene expression. Residue Tyr591 at the end of helix $\alpha \mathrm{G}$ is only about a distance of $16 \AA$ from Thr 480 on helix $\alpha \mathrm{C}$. These observations imply that it is likely that the very C-terminal end of the SEFEX module could wind back to approach the close vicinity of helix $\alpha \mathrm{C}$, together forming a composite ligand-binding surface that is necessary for recruiting the adaptor protein Act1. Further structural studies will be necessary to provide a more in-depth understanding.

Although it has previously been reported that IL-17RC also requires a 20-amino-acid C-terminal extension to its SEFIR domain for function (Ho et al., 2010), we believe that the intracellular signaling module of IL-17RC does not contain any extra functional motifs beyond its SEFIR domain. Ho and coworkers showed that truncation after residue 658 abolished IL-17 signaling, while constructs extending to at least residue 668 retained full function (Ho et al., 2010). However, our structure-based sequence alignment indicates that the true C-terminal boundary for the IL-17RC SEFIR domain lies at around residue 663 (Zhang et al., 2013). Therefore, truncation at residue 658 would cause partial deletion of the helix $\alpha \mathrm{E}$ from the IL-17RC SEFIR domain, perturbing its structural integrity. However, the construct extending to residue 668 would retain the complete SEFIR structural module that is necessary for signaling.

Presumably owing to limited proteolysis treatment (\$2), the fragment (residues 541-555 between helices $\alpha \mathrm{E}$ and $\alpha \mathrm{E}^{\prime}$ ) connecting the SEFIR domain and its C-extension is not visible in the structure of IL-17RA SEFIR. Our modeling showed that this missing region contains a short helix $(\alpha \mathrm{M})$ that is tightly packed against helices $\alpha \mathrm{A}$ and $\alpha \mathrm{E}$ (Fig. 5). It has previously been reported that a valine-to-histidine mutation in this helix (V550H) disrupted IL-17RA function (Maitra et al., 2007), and this residue was therefore inferred to be a key functional residue. The current structural data suggest that this defective mutant is presumably owing to perturbation of the stability and structural integrity of IL-17RA. Specifically, residue Val550 is completely buried inside the protein and makes extensive hydrophobic contacts with three aromatic residues, Phe401 $(\alpha \mathrm{A})$, Phe529 $(\alpha \mathrm{E})$ and Tyr558 $\left(\alpha \mathrm{E}^{\prime}\right)$, playing a structural role rather than being involved in protein-protein interactions (Fig. 5).

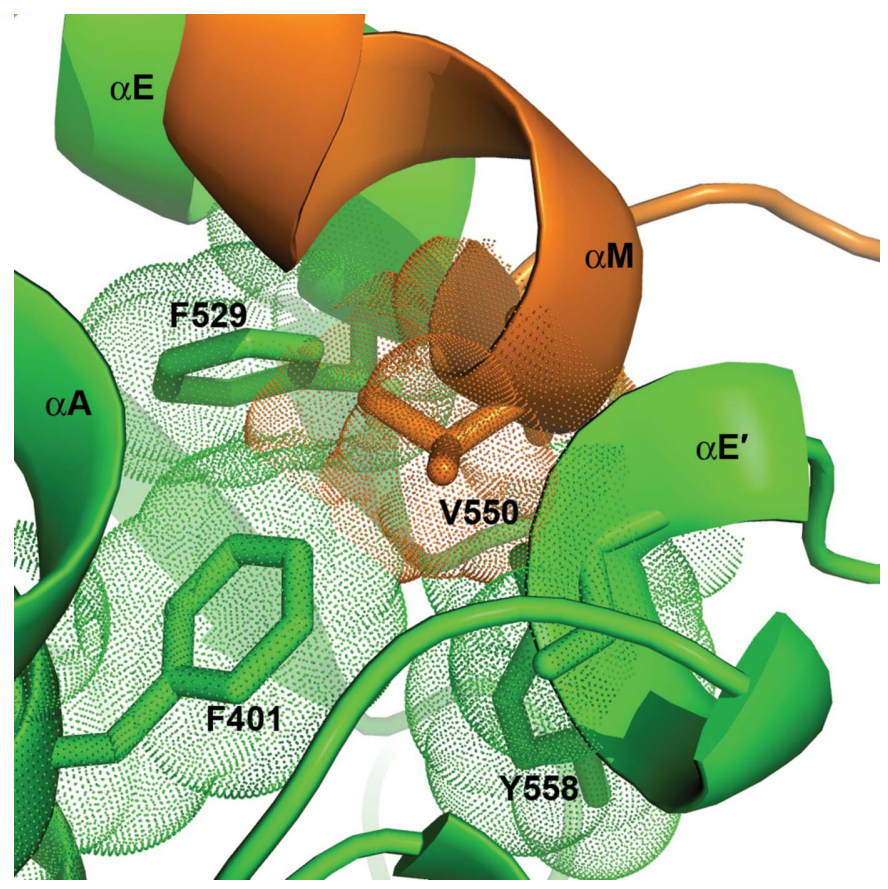

Figure 5

Val550 is a buried internal residue in IL-17RA SEFIR. The missing fragment (residues 541-555) connecting helices $\alpha \mathrm{E}$ and $\alpha \mathrm{E}^{\prime}$ of the crystal structure was modeled using FALC-Loop (http://falc-loop.seoklab.org/). Residue Val550 is located on helix $\alpha \mathrm{M}$ and is buried inside the protein, making extensive hydrophobic contacts with three aromatic residues from helices $\alpha \mathrm{A}$ (Phe401), $\alpha \mathrm{E}$ (Phe529) and $\alpha \mathrm{E}^{\prime}$ (Tyr558). The V550H mutation will disrupt the structural integrity. 
We thank the staff of beamline 19ID at the Advanced Photon Source for their support. We also thank Xiaolan Zhao (Genomics Core, Lerner Research Institute, Cleveland Clinic, Ohio, USA) for plasmid sequencing. JD is supported by NIH grants AI081928 and by the Oklahoma Agricultural Experiment Station at Oklahoma State University under project OKL02848. XL is supported by a Senior Investigator Award from the American Asthma Foundation and NIH grants R01HL098935-01A1 and R01NS071996. All authors declare no conflict of interest.

\section{References}

Adams, P. D. et al. (2010). Acta Cryst. D66, 213-221.

Chang, S. H. \& Dong, C. (2011). Cell. Signal. 23, 1069-1075.

Chang, S. H., Park, H. \& Dong, C. (2006). J. Biol. Chem. 281, 3560335607.

Chang, S. H., Reynolds, J. M., Pappu, B. P., Chen, G., Martinez, G. J. \& Dong, C. (2011). Immunity, 35, 611-621.

Chen, V. B., Arendall, W. B., Headd, J. J., Keedy, D. A., Immormino, R. M., Kapral, G. J., Murray, L. W., Richardson, J. S. \& Richardson, D. C. (2010). Acta Cryst. D66, 12-21.

Claudio, E., Sønder, S. U., Saret, S., Carvalho, G., Ramalingam, T. R., Wynn, T. A., Chariot, A., Garcia-Perganeda, A., Leonardi, A., Paun, A., Chen, A., Ren, N. Y., Wang, H. \& Siebenlist, U. (2009). J. Immunol. 182, 1617-1630.

Conti, H. R., Shen, F., Nayyar, N., Stocum, E., Sun, J. N., Lindemann, M. J., Ho, A. W., Hai, J. H., Yu, J. J., Jung, J. W., Filler, S. G., MassoWelch, P., Edgerton, M. \& Gaffen, S. L. (2009). J. Exp. Med. 206, 299-311.

DeLano, W. L. (2002). http://www.pymol.org.

Deng, J., Davies, D. R., Wisedchaisri, G., Wu, M., Hol, W. G. J. \& Mehlin, C. (2004). Acta Cryst. D60, 203-204.

Emsley, P., Lohkamp, B., Scott, W. G. \& Cowtan, K. (2010). Acta Cryst. D66, 486-501.

Gaffen, S. L. (2009). Nature Rev. Immunol. 9, 556-567.

Gonzalez-García, I., Zhao, Y., Ju, S., Gu, Q., Liu, L., Kolls, J. K. \& Lu, B. (2009). J. Immunol. 182, 2665-2671.

Ho, A. W., Shen, F., Conti, H. R., Patel, N., Childs, E. E., Peterson, A. C., Hernández-Santos, N., Kolls, J. K., Kane, L. P., Ouyang, W. \& Gaffen, S. L. (2010). J. Immunol. 185, 1063-1070.
Hu, Y., Shen, F., Crellin, N. K. \& Ouyang, W. (2011). Ann. N. Y. Acad. Sci. 1217, 60-76.

Iwakura, Y. \& Ishigame, H. (2006). J. Clin. Invest. 116, 1218-1222.

Krumm, B., Meng, X., Li, Y., Xiang, Y. \& Deng, J. (2008). Proc. Natl Acad. Sci. USA, 105, 20711-20715.

Leonardi, A., Chariot, A., Claudio, E., Cunningham, K. \& Siebenlist, U. (2000). Proc. Natl Acad. Sci. USA, 97, 10494-10499.

Liu, C. et al. (2011). Sci. Signal. 4, ra72.

Maitra, A., Shen, F., Hanel, W., Mossman, K., Tocker, J., Swart, D. \& Gaffen, S. L. (2007). Proc. Natl Acad. Sci. USA, 104, 75067511.

McCoy, A. J., Grosse-Kunstleve, R. W., Adams, P. D., Winn, M. D., Storoni, L. C. \& Read, R. J. (2007). J. Appl. Cryst. 40, 658-674.

Minor, W., Cymborowski, M., Otwinowski, Z. \& Chruszcz, M. (2006). Acta Cryst. D62, 859-866.

Nakae, S., Komiyama, Y., Nambu, A., Sudo, K., Iwase, M., Homma, I., Sekikawa, K., Asano, M. \& Iwakura, Y. (2002). Immunity, 17, 375-387.

Novatchkova, M., Leibbrandt, A., Werzowa, J., Neubüser, A. \& Eisenhaber, F. (2003). Trends Biochem. Sci. 28, 226-229.

Onishi, R. M., Park, S. J., Hanel, W., Ho, A. W., Maitra, A. \& Gaffen, S. L. (2010). J. Biol. Chem. 285, 32751-32759.

Painter, J. \& Merritt, E. A. (2006). Acta Cryst. D62, 439-450.

Papp, K. A., Leonardi, C., Menter, A., Ortonne, J. P., Krueger, J. G., Kricorian, G., Aras, G., Li, J., Russell, C. B., Thompson, E. H. \& Baumgartner, S. (2012). N. Engl. J. Med. 366, 1181-1189.

Qian, Y. et al. (2007). Nature Immunol. 8, 247-256.

Ramirez-Carrozzi, V. et al. (2011). Nature Immunol. 12, 11591166.

Schnyder-Candrian, S., Togbe, D., Couillin, I., Mercier, I., Brombacher, F., Quesniaux, V., Fossiez, F., Ryffel, B. \& Schnyder, B. (2006). J. Exp. Med. 203, 2715-2725.

Song, X., Zhu, S., Shi, P., Liu, Y., Shi, Y., Levin, S. D. \& Qian, Y. (2011). Nature Immunol. 12, 1151-1158.

Swaidani, S., Bulek, K., Kang, Z., Liu, C., Lu, Y., Yin, W., Aronica, M. \& Li, X. (2009). J. Immunol. 182, 1631-1640.

Van Duyne, G. D., Standaert, R. F., Karplus, P. A., Schreiber, S. L. \& Clardy, J. (1993). J. Mol. Biol. 229, 105-124.

Ye, P., Garvey, P. B., Zhang, P., Nelson, S., Bagby, G., Summer, W. R., Schwarzenberger, P., Shellito, J. E. \& Kolls, J. K. (2001). Am. J. Respir. Cell Mol. Biol. 25, 335-340.

Zhang, B., Liu, C., Qian, W., Han, Y., Li, X. \& Deng, J. (2013). J. Immunol. 190, 2320-2326. 